\title{
Attribution Des Noms Et Pratiques En Santé Materno-Infantile : Approche Socio-Anthropologique Des Enfants Sacrés En Pays Abouré Et Baoulé De Cote D'ivoire
}

\section{Sylvie N'Goran}

Unité de Formation et de Recherche (UFR), Communication Milieu et Société (CMS), Département d'Anthropologie et de Sociologie (DAS), Université Alassane Ouattara (UAO), Bouaké, Côte d’Ivoire

\section{Maxime Oga}

Unité de Formation et de Recherche (UFR), Communication Milieu et Société (CMS), Département d'Anthropologie et de Sociologie (DAS), Université Alassane Ouattara (UAO), Bouaké, Côte d'Ivoire PAC-CI (PNLS, AC $_{12}$, Coopération française - Côte d'Ivoire), CHU de Treichville, Site ANRS, Abidjan, Côte d'Ivoire

\section{doi: 10.19044/esj.2017.v13n11p182 URL:http://dx.doi.org/10.19044/esj.2017.v13n11p182}

\begin{abstract}
The birth of a child is considered as a joyous event and comes along with celebrations and festivities according to the tradition of each cultural group. The name given to the new-born may be that of a living grandparent, one of the parents, or a friend of the child's father. However, the name given may also be related to the birth order. The birth of a child, who is a fragile and vulnerable being, gives rise to indispensable rituals that takes into account its rank. These rituals are not only for its survival and development, but also for its socialization and the transformation of its identity. Tradition also involves cultural identity through name assigning in order to protect the child from all kinds of diseases. Moreover, traditional practices regarding the issue of child health serves as forms of protection, humanization, and integration of the child into the society. What about the Aboure and N'Zipkly people? From a comprehensive perspective on the specificity of the names given from birth, this study aims to grasp the plausible influences on child health based on the relationships between the child and his name as perceived by the Abouré and N'Zipkly people. To achieve this, we asked ourselves the following questions: Why does a name has a significant influence on the health of the newborn? What is the relationship between child health and name? What are the socio-cultural practices of sacred
\end{abstract}


children? Furthermore, there were various questions that led to data collection by observing activities and practices related to sacred children. The data collection was done using interview guides for mothers with children aged 0 to 5 years, grandparents, and traditional therapists.

Keywords: Name, Health, Maternal and Child, Childhood diseases, Traditional practices, Abouré, Baoulé, Côte d’Ivoire

\section{Resumé}

La naissance de l'enfant est perçue comme une joie et s'accompagne de célébration selon la tradition de chaque peuple. Le nom attribué au nouveau-né à la naissance peut-être celui d'un grand-parent vivant, des géniteurs, d'un ami du père de l'enfant. Mais l'attribution du nom peut être aussi fonction du rang de naissance. La naissance de l'enfant, être fragile et vulnérable, donne lieu à des rituels indispensables qui tiennent compte de son rang. Ces rituels sont non seulement indispensables à sa survie et à son développement mais aussi à sa socialisation et à la transformation de son identité. La tradition fait aussi appel à l'identité culturelle par l'attribution du nom pour protéger l'enfant contre toutes formes de maladie. Et, les pratiques traditionnelles sur la question de santé infantile sont des formes de protection, d'humanisation et d'intégration de celui-ci dans la société. Qu'en est-il dans les peuples Abouré et N'Zipkly? Dans une perspective compréhensive de la spécificité des noms attribués dès la naissance, nous avons pour objectif de saisir à partir des relations entre l'enfant et son nom telles que pensées par les peuples Abouré et N'Zipkly, les plausibles influences sur sa santé. Pour cela, nous nous sommes posé les questions suivantes : pourquoi attribuer un nom influence t-il la santé chez le nouveauné ? Quelles relations entre la santé de l'enfant et son nom ? Quelles sont les pratiques socioculturelles liées aux enfants sacrés ? Questionnement ayant conduit à une collecte de données par observation des activités et pratiques liées aux enfants sacrés à l'aide des guides d'entretien adressés aux mères ayant des enfants de zéro à cinq ans, aux grands-parents, et aux tradithérapeutes.

Mots clés : Nom, Santé, Materno-Infantile, Maladies infantiles, pratiques traditionnelles, Abouré, Baoulé, Côte d’Ivoire

\section{Introduction}

Dans certaines sociétés africaines, les enfants se distinguent par leur ordre de naissance. Les noms à leur attribuer en dépendent et donnent lieu pour certains à des rituels, des cérémonies sacrificielles mobilisant à la fois les familles du père, de la mère de l'enfant et même de toute la communauté 
(Mbiti, 1971). Le nom fait intégralement partie de la vie de l'enfant, dans toutes les formes de civilisations selon une règle bien établie, et le guide. L'attribution du nom, disent certains auteurs, contribue à la protection du nouveau-né et à son inscription dans le monde (Haxaire, 2007). Elle s'accompagne de cérémonies baptismales, de consécrations d'autels visant à améliorer sa santé et son bien-être. Forme de reconnaissance sociale, l'attribution du nom est un rite d'agrégation (Van, 1981) et par là même, un processus de distinction. De fait, dans l'usage quotidien, il y a des entités qui ont droit au nom propre et d'autres non. Ce qui conduit à avoir le groupe de ceux dont on connaît le nom propre et ceux chez qui il est ignoré. (Bromberger, 1982). Les soins materno-infantiles dépendent en grande partie du nom, en fonction du rang de naissance, que porte l'enfant ; et, chaque communauté par ses représentations socioculturelles, dont l'attribution du nom par ordre de naissance, s'attèle à procurer à l'enfant un meilleur être. En pays Abouré et Baoulé, la santé infantile dépend en grande partie de la relation que la communauté entretient avec le monde visible et le monde invisible dont les représentations autour de l'attribution du nom en sont une composante.

De façon concrète et selon les peuples, il existe un système de nomination préétabli basé sur l’ordre des naissances (Collard, 1973) comme c’est le cas chez les Abouré de Bonoua et les Baoulé (N'Zikply) de Didiévi. Les noms propres peuvent aussi classer les individus selon leur origine et leur statut social, mais selon diverses formes. Dans nos sociétés, la simple consonance d'un nom renseigne approximativement au moins sur l'origine ethnique ou régionale de son porteur (Bromberger, 1982). Le nom s'avère non seulement une adresse sociale mais plus encore un facteur d'identification et d'intégration au groupe socioculturel (Kasongo, 1988). Chaque peuple (Abouré, Baoulé) est caractérisé par des noms qu’il attribue à ses enfants avec un traitement particulier que bénéficient certains d'entre eux qui sont dits « enfants sacrés ». Si, en pays Abouré les enfants dits "sacrés » se définissent comme les enfants jumeaux, post-jumeaux et ceux occupant les $3^{\text {ème }}, 4^{\text {ème }}, 7^{\text {ème }}, 8^{\text {ème }}, 9^{\text {ème }}$ et $10^{\text {ème }}$ rangs de naissance, ce n'est pas le cas en pays Baoulé N’Zikply. Chez le N’Zikply, peuvent être classés parmi les « enfants sacrés » : les enfants jumeaux, post-jumeaux et ceux occupant les $3^{\text {ème }}$ et $10^{\text {ème }}$ rangs de naissance.

Si pour Claude Lévi-Strauss, le nom est universel et sert, dans toutes les sociétés, à signifier, à identifier, à classer (Lévi-Strauss, 1962), dans cet article, nous comptons comprendre à partir de la relation existant entre l'enfant et son nom, les plausibles influences sur sa santé. La question que nous posons est la suivante : pourquoi attribuer un nom influence t-il la santé chez le nouveau-né ? Quelles relations entre la santé de l'enfant et son nom? Quelles sont les pratiques socioculturelles liées aux enfants sacrés? 
Pour y parvenir, nous avons collecté nos données par observation des activités et pratiques liées aux enfants sacrés et à l'aide des guides d'entretien (un focus-group et un entretien semi-directif) adressés aux mères ayant des enfants de zéro à cinq ans, aux grands-parents, et aux tradithérapeutes. Deux grandes parties structurent cet article à savoir : 1) l'univers représentationnel de l'enfant qui tient compte de comment on donne le nom au nouveau-né dans les peuples Abouré et Baoulé; 2) l’impact socio-sanitaire de cet univers représentationnel sur la santé de l'enfant qui aborde à la fois les exemples des « enfants sacrés », des pratiques sanitaires et des rituels qui leur consacrent une santé et un bien être.

\section{Résultats}

\section{Univers représentationnel de l'enfant en pays Abouré et Baoulé}

Il se caractérise dans cet article par l'attribution du nom dans ces deux peuples.

\section{Les noms en fonction du rang de naissance \\ Chez les Abouré de Bonoua}

En pays Abouré, l’attribution du nom de l'enfant commence avec la naissance du troisième enfant du même sexe né de la mère. Ici, la descendance de l'homme n'est pas prise en compte. Les troisièmes enfants sont appelés Missan quand ils sont de sexe masculin et Massan quand ils sont du sexe féminin. Le quatrième enfant de sexe opposé s’appelle Aprâh et celui ayant le même sexe s'appelle Nindin. Le septième enfant, du même sexe que les trois premiers, de sexe féminin ou masculin s’appelle Assouan. Le huitième enfant s’appelle Yamouké, Niangoran ou Yangra est le neuvième enfant de sexe féminin ou masculin, Obrou, le dixième enfant.

Tableau 1. Noms attribués en pays Abouré de Bonoua aux enfants sacrés

\begin{tabular}{|c|c|c|c|c|c|c|}
\hline \multicolumn{7}{|c|}{ Noms attribués en pays Abouré de Bonoua aux enfants sacrés } \\
\hline Sexe & $3^{\text {eemes }}$ enfants & $\begin{array}{c}4^{\text {emes }} \\
\text { enfants }\end{array}$ & $\begin{array}{c}7^{\text {ėmes }} \\
\text { enfants }\end{array}$ & $\begin{array}{c}8^{\text {emes }} \\
\text { enfants }\end{array}$ & $\begin{array}{c}9^{\text {emes }} \\
\text { enfants }\end{array}$ & $\begin{array}{c}10^{\text {èmes }} \\
\text { enfants }\end{array}$ \\
\hline Masculin & Missan & $\begin{array}{c}\text { Aprâh ou } \\
\text { Nindin }\end{array}$ & Assouan & Yamouké & $\begin{array}{c}\text { Niangoran } \\
\text { ou Yangra }\end{array}$ & Obrou \\
\hline Féminin & Massan & $\begin{array}{c}\text { Aprâh ou } \\
\text { Nindin }\end{array}$ & Assouan & Yamouké & $\begin{array}{c}\text { Niangoran } \\
\text { ou Yangra }\end{array}$ & Obrou \\
\hline
\end{tabular}

Source: Données d'enquête, Larabia, Sous-préfecture de Bonoua, Côte d’Ivoire

\section{Chez les baoulés de Didiévi (Baoulé N’Zikply)}

Les noms ou les post noms sont les mêmes selon le sexe qu'on soit du sexe masculin ou féminin. Le troisième enfant du même sexe que les deux premiers est appelé Hissan ( $N$ 'Guessan) et le troisième enfant de sexe opposé aux deux autres premiers s'appelle Kindô En pays Baoulé, N’Dri est 
le nom attribué au quatrième enfant de sexe identique aux trois premiers enfants quand le neuvième enfant est appelé $N^{\prime}$ Goran, le dixième Brou, le onzième Loukou, le douzième Djanh, le treizième $N^{\prime}$ Gbain. Les jumeaux sont appelés N’Da et l'enfant qui nait après les jumeaux est appelé Amani.

L'attribution des noms par ordre de naissance est d'une importance chez le Baoulé N'Zikply parce que participant au bien-être des enfants. Les Baoulés de Didiévi croient que certains enfants, en fonction de leur ordre de naissance, ont une âme dangereuse quand d'autres ont une âme bienveillante. Parmi les enfants précités, les plus sacrés sont les troisièmes, dixièmes, les jumeaux $\left(N^{\prime} D a\right)$ et les enfants nés après les jumeaux, les post-jumeaux (Amani). Pour chacun de ces enfants, des cérémonies sacrificielles et des rituels sont requis.

Tableau 2. Noms attribués en pays N’Zikply de Didiévi aux enfants sacrés

\begin{tabular}{|c|c|c|c|c|}
\hline \multicolumn{5}{|c|}{ Noms attribués en pays N'Zikply de Didiévi aux enfants sacrés } \\
\hline Sexe & $3^{\text {èmes }}$ enfants & $10^{\text {emes }}$ enfants & Jumeaux & Post-jumeaux \\
\hline Masculin & Hissan ou N'Guessan & Brou & N'Da-Bian & Amani \\
\hline Féminin & Hissan ou N'Guessan & Brou & N'Da-Bla & Amani \\
\hline
\end{tabular}

Source: Données d'enquête, Didiévi, Sous-préfecture de Yamoussoukro, Côte d’Ivoire

\section{Impact socio-sanitaire de l'univers représentationnel de l'enfant sur sa santé à Bonoua et Didiévi}

En pays Abouré comme c’est aussi le cas en pays N'Zikply, à l'endroit des enfants sacrés, des précautions sociales et sanitaires sont prises pour leur éviter de tomber malade.

\section{Chez les abouré de Bonoua}

Selon l'ordre de naissance, à compter du troisième enfant, des colliers sont confectionnés pour les enfants et des rituels sont faits. Pour le troisième enfant du même sexe, Missan (de sexe masculin) et Massan (de sexe féminin), Bamon-fêh (Figure 1) est le collier confectionné et mis à leur poignet. Le collier est réputé agir contre les fortes fièvres que pourraient faire l'enfant.

«Chez nous les abourés, si tu as un troisième enfant de même sexe que les deux premiers, tu dois lui porter le collier et faire le rituel qu'il faut pour éviter qu'il fasse une forte fièvre, qui nécessite une consultation chez la thérapeute du village». Mlle Kissy Véronique, Mère d'un troisième enfant du même sexe, célibataires, âgées de 46 ans, Larabia, Sous-préfecture de Bonoua. 
Figure 1. Bamon-fêh

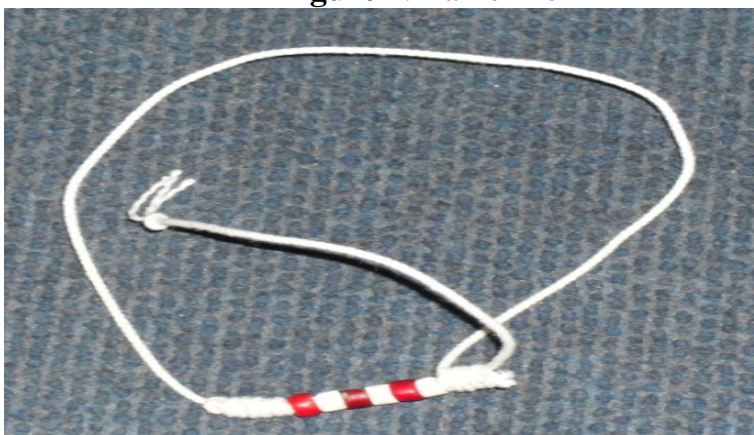

Source: Données d’enquête, Larabia, Sous-préfecture de Bonoua, Côte d’Ivoire

Bamon-fêh est aussi d'usage chez le quatrième enfant de sexe opposé ainsi que chez les septièmes, huitièmes, neuvièmes et dixièmes enfants. La confection du collier Bamon-fêh tient compte du rang de naissance de l'enfant, devant le porter, et exige que le nombre de perles utilisées soit proportionnel au rang de naissance. Enfilées de cette façon, les trois perles rouges, figure 1 susmentionnée, représentent les trois enfants successifs de même sexe et les deux perles blanches attestent que les trois enfants de mêmes sexes sont nés du même père et de la même mère. Après sa confection, faire porter le Bamon-fêh à l'enfant servirait à le protéger des éventuelles dermatoses et de toute déformation.

Aprâh, quatrième enfant de sexe opposé porte à lui seul deux colliers dont un au poignet (Bamon-fêh) et l'autre au cou (Aprâh-fêh). Il se compose d'une perle de couleur bleue au centre de la file de perles pour marquer une différence entre le quatrième enfant et les trois autres, de même sexe, représentés par les deux autres perles blanches situées aux deux extrémités de la file. Cette disposition particulière montre le lien fraternel unissant les trois enfants au quatrième. Pour Aprâh, un rituel de lavage du visage lui est ensuite fait pour lui éviter d'être soit attardé, soit violent soit encore chétif. Tout quatrième enfant de sexe opposé ou Aprâh, en plus de protéger sa santé par Bamon-fêh, le collier à son poignet, il se munit d'un autre collier au cou qu'on appelle Aprâh-fêh (figure 2) pour sa protection contre les mauvais esprits. Porté, Aprâh-fêh permet aussi de distinguer le rang de naissance de l'enfant dans une famille donnée.

"Nous portons le Bamon-fêh au bras et Aprâh-fêh au cou de l'enfant ; Nous faisons un canari pour éviter que l'enfant ait soit le corps chaud ou soit atteint de folie à la longue » Mme Adiko, Tradipraticienne, 60 ans, Larabia, Sous-préfecture de Bonoua. 
Figure 2: Aprâh-fêh

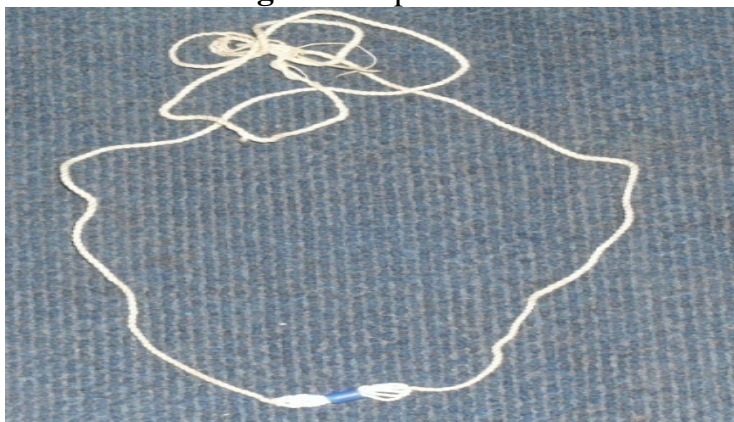

Source: Données d’enquête, Larabia, Sous-préfecture de Bonoua, Côte d’Ivoire

Le septième enfant, de sexe masculin ou féminin, s’appelle Assouan. Il est considéré comme un enfant béni et le port de son collier Bamon-fêh rappelle sa position ordinale de naissance.

« Le septième enfant est considéré comme la clé qui ouvre la porte aux enfants bénis, porte les deux colliers pour éviter les mauvais sorts » Mme Adiko, Tradipraticienne, 60 ans, Larabia, Sous-préfecture de Bonoua.

Figure 3: Agblêh-fêh

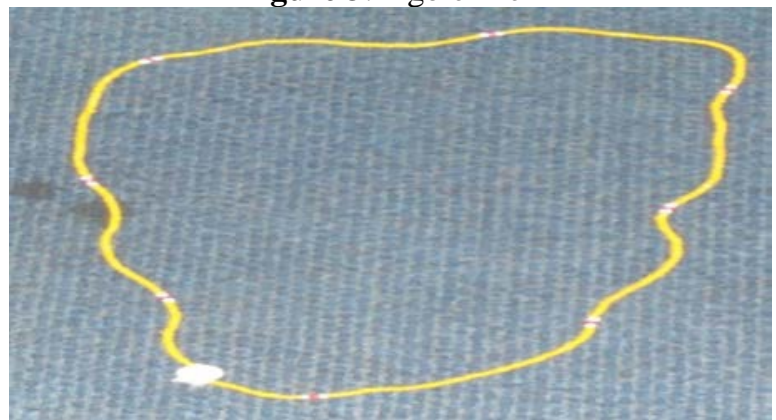

Source: Données d'enquête, Larabia, Sous-préfecture de Bonoua, Côte d’Ivoire

Agblêh-fêh se porte au cou du septième, huitième, neuvième, dixième enfant et des jumeaux. La perle de couleur blanche au milieu rappelle que l'enfant est béni. Assemblées, les perles de couleur blanche et rouge enfilés en collier confirment le statut sacré et béni de tout enfant qui le porte. Mais le port de ce collier, Agblêh-fêh, devra se faire après un rituel de droit au marigot en l'honneur de l'enfant qui porte par la suite le collier.

Yamouké, le huitième enfant bénéficie d'une cérémonie spéciale parce qu'il possède des pouvoirs sacrés. Pour lui donc, sa mère doit se rendre avec lui au marigot pour un bain suivi d'un rituel à la fin duquel on lui porte deux colliers dont un à son bras (Bamon-fêh) et l'autre à son cou (Agblêhfêh).

« Le huitième enfant a droit à une cérémonie spéciale et importante parce qu'il est sacré à cause de ses pouvoirs sacrés ; 
sans le rituel et le port de collier, celui-ci est victime de la paralysie au niveau des pieds ». Mme Vanga Françoise, Tradipraticienne, 60 ans, Larabia, Sous-préfecture de Bonoua.

Le neuvième enfant de sexe masculin ou féminin s’appelle Niangoran ou Yangra et a droit à la même cérémonie que le huitième enfant ; le port des différents colliers (Bamon-fêh au bras et Agblêh-fêh au cou) le protège de la paralysie et de la déstabilisation sociale.

« Nous reconnaissons le huitième et le neuvième enfant par les vêtements qu'ils mettent. Le neuvième enfant est aussi sacré parce qu'il est aussi exposé à la paralysie et à la déstabilisation sociale ». Mme Wognin, Mère d'un huitième enfant, mariée, 57 ans, Bonoua.

Obrou est le dixième enfant et a droit à toutes les cérémonies. « Le dixième enfant est sacré et spécial. Il fait valoir ses droits lors de la cérémonie de la présentation du dixième enfant ; il est exposé à la sorcellerie » Prophétesse 65 ans, Assè, Souspréfecture de Bonoua.

Figure 4. Manmouma

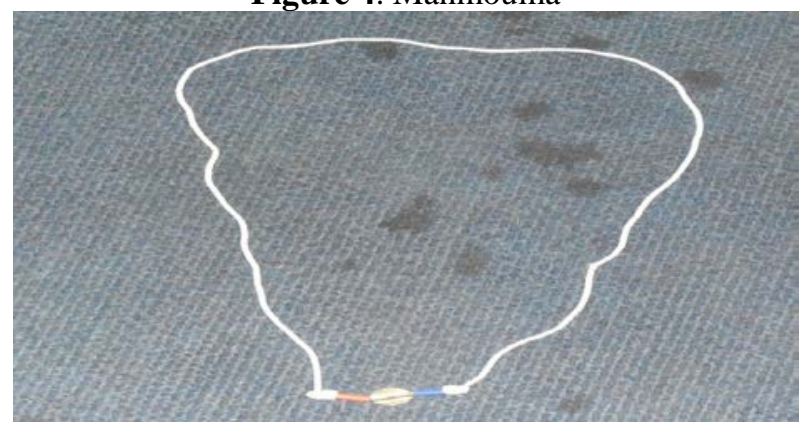

Source: Données d’enquête, Larabia, Sous-préfecture de Bonoua, Côte d’Ivoire

Manmouma-fêh porté au cou protège la santé des enfants suivants: les jumeaux, les septièmes aux dixièmes enfants. Les perles de couleur bleue et rouge font savoir que ces enfants sacrés ont eu droit à un rituel et une cérémonie en leur honneur ; quand la perle de couleur argent qui se situe entre les deux perles de couleur bleue et rouge renseigne sur le caractère sacré et béni des enfants qui les portent. Par ailleurs, Manmouma-fêh ne se porte jamais seul et est toujours associé à Agblêh-fêh.

Les enfants jumeaux droits (jumeaux de même sexe) sont appelés $N$ 'Tatrinlin et les enfants jumeaux noirs (jumeaux de sexes opposés) appelés N'Tabi. Les enfants jumeaux N'Tatrinlin et N'Tabi prennent un bain au marigot suivi d'un rituel avec une cérémonie appelé Djanchô au domicile des parents des enfants à la suite de laquelle des colliers spécifiques leur sont portés pour éviter la sorcellerie. 
«Les enfants jumeaux de sexe masculin s'appellent pour le premier Wognin et le second Vanga; et, les enfants jumeaux de sexe féminin s'appelle pour la première Atwo et la deuxième Wouman. Ils prennent un bain au marigot suivi de rituel avec la cérémonie de 'Djanchô' à la maison. Ils portent des colliers spécifiques pour qu'il ait la maîtrise de soi, la douceur et la tranquillité de l’âme, éviter la sorcellerie ». Mme Kodia, Mère d'enfants jumeaux, 45 ans, mariée, Larabia, Bonoua.

En pays Abouré, les enfants sont dits sacrés lorsqu'ils respectent les exigences cumulatives suivantes. Premièrement, ils doivent être nés à partir du troisième enfant du même sexe que les trois premiers et issus de la même mère. Deuxièmement, ces enfants là doivent être des jumeaux ou encore selon le rang de naissance être : troisième, quatrième, septième, huitième, neuvième et dixième. Ces enfants sacrés portent des noms ou post-noms qui nécessitent des cérémonies et rituels appropriés pour les mettre à l'abri des maladies incurables naturelles ou provoquées par des humains ou des mauvais esprits. En prévision d'un état de bonne santé chez l'enfant, ces cérémonies se font pour le protéger contre les sorts, les mauvaises langues et des éventuelles actions des sorciers et des mauvais esprits. Le rang de naissance de l'enfant de même que le type d'enfant né (naissance gémellaire ou non) en pays Abouré requiert des noms précis couplés à des pratiques particulières (cérémonies, rituels, port des colliers, usage des décoctions faites de diverses plantes et écorces médicinales) qui garantissant à l'enfant un meilleur état de santé physique, psychologique, mental et social. Qu'en est-il chez les baoulés N’Zipkly de Didiévi?

\section{Chez les baoulés de Didiévi}

Les prénoms pour un nouveau-né de sexe masculin ou de sexe féminin sont les mêmes. Les enfants par leur ordre de naissance sont considérés comme ayant chacun soit une bonne âme soit une mauvaise.

Particulièrement, le troisième de même sexe que les deux premiers enfants, appelé Hissan ( $N$ 'Guessan), est considéré comme ayant une âme à la fois bienveillante et malveillante. Pour ce nom qu'il porte et pour son état de santé, leurs parents selon les dispositions coutumières devraient le conduire chez le devin. Dans la pratique, le devin recommande pour Hissan l'instauration d'un autel en son honneur pour restaurer son bien-être. La construction de cet autel met à contribution le Nofani de forme variable en fonction de l'usage: il peut-être soit long soit rond. Le Nofani est un médicament fait à base soit de terre soit d'un ensemble de feuilles et d'écorces soit de kaolin. Le Nofani est supposé être, déposé dans une petite cuvette au chevet de l'enfant. Ici, sous la forme de kaolin, le Nofani servirait à apaiser l'âme de l'enfant quand la forme composée de feuilles et d'écorces 
aide à chasser les mauvais esprits. Il revient à la mère de faire usage du Nofani qui se présente sous une forme volumineuse. Ainsi présenté, la mère utilise le Nofani en le passant sur le corps de l'enfant de sorte à l'en couvrir jusqu'à rien avoir en reste. Cette façon de faire servirait à la protection et à la procuration du bonheur à l'enfant. Âme malveillante aussi, pour Hissan il faut accomplir un rituel d'apaisement, de purification. Hissan se caractériserait par un état de santé précaire marqué par de fortes fièvres et migraines régulières.

"Hissan est quelqu'un de "chaud et brûlant", (turbulent, brouillon) très capricieux. Hissan a la bougeotte, touche à tout, toujours en mouvement et rapide dans ses actions. Pour Hissan yèyè (N'Guessan brûlant), le rituel de lavage du visage atténue les fortes fièvres et les fortes migraines ». Mme Brou, Grandmère, 56 ans, Didiévi.

Le troisième enfant de sexe opposé aux deux premiers s'appelle Kindôh. C'est un enfant très turbulent, insolent et très têtu. Son rituel de bain de lavage est constitué d'un mélange d'eau, de kaolin et de feuilles. L'eau est symbole de la pureté, le kaolin calme l'ardeur de l'âme et les feuilles chassent les esprits nuisibles à son bien-être ; composition qui participe à l'apaisement de l'âme de Kindôh afin de lui permettre une vie harmonieuse avec la communauté.

« Kindôh est l'enfant qui marque une rupture de la maternité ; la mère a pour obligation morale à continuer de procréer. » $\mathrm{Mr}$ Koffi Isidore, Thérapeute, 60 ans, Didiévi.

$N$ 'Dri est le quatrième enfant de sexe identique aux trois premiers. $N$ 'Dri est l'enfant que toute famille ou tout couple souhaiterait avoir parce que bénéficierait d’une représentation socialement située qui le crédite d'avoir la capacité d'influencer le cours de la vie dans son environnement en y apportant l'harmonie familiale et sociale. Il répond aux principes d'ordre social dont l'harmonie familiale et sociale. $N$ 'Dri est de ce fait chanceux, clairvoyant avec la fonction de pouvoir changer le cours des choses, transformer le cours de la structure famille dont il est issue. Pour lui donc, un rituel de lavage de visage ayant les mêmes constituants que le rituel de bain de lavage de Kindôh ; c’est-à-dire, un mélange d'eau, de kaolin et de feuilles qui servirait à lui éviter les pathologies ophtalmologiques et les mauvais sort.

« N’Dri est un enfant doux et remplit de joie ; le rite de lavage de visage protège l'enfant contre les problèmes d'yeux et de la méchanceté des hommes. » Mme Kouassi, Tradipraticienne 58 ans, Didiévi.

N’Goran, neuvième enfant, est considéré comme un enfant clairvoyant et compliqué. Il est caractériel, calme et très silencieux. N’Goran a une âme apaisée et il est moins turbulent. En principe maladif, il revient à 
ses parents dans une perspective préventive d'avoir recours au devin qui bâtira un autel ayant les mêmes caractéristiques que celui construit pour Hissan en suivant les mêmes applications. Le rituel consiste à éviter des maladies telles que l’épilepsie, la démangeaison et le corps chaud.

« N’Goran est un enfant comme le troisième, il est compliqué.

On pratique le rituel de lavage de visage pour ne pas qu'il ait

l’épilepsie » Mr Kouassi Yao, Thérapeute, 60 ans, Didiévi.

Le dixième enfant est appelé Brou. Il est lui-même sacré et exceptionnel. Il est exceptionnel parce qu'à sa naissance, il est donné en adoption définitive à sa famille maternelle, plus précisément à son oncle du côté maternelle. Cela participe à solidifier les liens entre les deux familles de l'enfant. En contre partie à ce don du dixième enfant, l'oncle maternel offre un mouton à son beau-frère, symbole de reconnaissance, de joie et de remerciement. La sacralité de l'enfant Brou reste aussi marquée par la consécration d'un autel que construisent les parents et qui est aussi semblable à celui fait pour Hissan ou N'guessan. Sa sacralité et son exceptionnalité s'expliquent aussi par ce que Brou est parfois objet d’adoration précisément, lorsque certaines personnes déposent dans un récipient placé à côté de l'autel qui lui est dédié des pièces d’argent en faisant des prières pour la réalisation de leurs vœux. De même que Hissan ( $N$ 'guessan) et $N^{\prime}$ Goran, l'autel consacré à Brou le protège aussi de la sorcellerie, des pathologies ophtalmologiques, et des conséquences des mauvaises langues.

« Le dixième enfant est spécial car il symbolise l’unité entre les deux familles maternelle et paternelle. À la naissance de Brou, on dépose un canaris dans la maison pour le protéger contre la sorcellerie ». Mme Kouakou, Grand-mère, 57 ans, Mariée,

Didiévi.

Le onzième enfant est appelé Loukou, le douzième enfant Djanh, et le treizième enfant $N^{\prime}$ Gbain. Ce sont tous des enfants sacrés. En pratique à leur naissance, ils sont objets d'un rituel avec comme composants l'eau et le kaolin pour les purifier et leur procurer la joie, la paix. L'eau symbolise la pureté quand le kaolin garantie l'assurance à l'âme. Loukou, Djanh et $N$ 'Gbain souffrent le plus souvent des pathologies suivantes : mal de ventre, céphalées, forte migraine et les pathologies ophtalmologiques. Pour les en protéger, un rituel de lavage du visage les purifiant est exigé et auquel ces enfants se soumettent après que leurs parents aient pris toutes les dispositions requises à savoir la consultation d'un guérisseur traditionnel, le sacrifice d'un poulet africain de couleur blanche et un œuf de poulet africain.

«Loukou, Djanh et N'Gbain sont des enfants dotés de don de clairvoyance. Ces enfants sont à la fois clairvoyants, durs de caractère et têtus. Très souvent, ils souffrent de douleurs de ventre, de forte 
migraine et de maux d'yeux; Il faut faire le sacrifice de poulet et de cabri pour qu'ils soient en bonne santé ». Mr Kouadio Kouadio, Thérapeute, 60 ans, Didiévi.

Les jumeaux sont appelés $N$ 'Da et sont des enfants craints et honorés parce que dotés de pouvoirs qui doivent être fortifiés et entretenus par l'instauration d'un autel. Dès leur naissance, les parents déposent deux œufs dans un endroit de la maison pour symboliser les esprits des jumeaux. Sous la prescription du devin, l'instauration de l'autel s'accompagne de sacrifice composé d'un mouton, de deux ou quatre poulets pour symboliser et marquer la joie partagée avec toute la communauté par la fabrication de "SèkèSèkè ». Les $N^{\prime} D a$ sont objets d'adoration matérialisée par des œufs à côtés desquels les parents rangent deux petits récipients servant de réceptacles de pièces d'argents déposées après un vœu, un souhait. Pour eux, chaque fin d'année, les parents font du " afoliè ${ }^{44}$ " qu'ils déposent à cet autel qui leur est dédié. Un rite qui permettrait de demander le bonheur et la protection des jumeaux et de la famille. Particulièrement, ces cérémonies sacrificielles protègent les jumeaux de la sorcellerie et de la colère de leur génie protecteur.

«Le jour de leur sortie, les parents font confectionner des objets de danse appelés "Sèkè- Sèkè" pour les jumeaux ; c’est un objet important parce qu'ils sont des enfants sorciers et pour éviter de les mettre en colère " Mme Kouamé, Mère de jumeaux, 47ans, Mariée, Langui-kouadiokro.

L'enfant né après les jumeaux s'appelle Amani et est considéré comme guide des jumeaux. Il partage, avec les jumeaux qu'il suit, presque : soins sanitaires en cas de maladie, autel d'adoration. Amani est un enfant sacré parce qu'après sa naissance, il réclame aussi la naissance de post jumeaux. Ce qu'il manifeste par la survenue de maladies diverses et donc un mauvais état de santé répété. Dans ce cas, ses parents pour ses soins se servent du même traitement que pour les jumeaux parce qu'il est leur protecteur et leur défenseur mais bien plus, les jumeaux partagent tout ce qui leur revient avec leur Amani. En cas de survenue de pathologies graves ou pas comme le corps chaud, le paludisme, et la fièvre, les parents d'Amani consultent toujours un devin pour une solution thérapeutique appropriée vue son caractère d'enfant sacré.

«Amani est le guide des jumeaux parce qu'il est clairvoyant et a une âme guerrière. En cas de décès des jumeaux, l'autel des jumeaux est attribué à Amani pour le protéger de la sorcellerie et

\footnotetext{
${ }^{44}$ Afoliè ou encore foufou igname est un met fait à base de tubercule d'igname rendue patte après écrasement et avec ajout de l’huile de palme.
} 
du mauvais sort» Mlle Yao Amenan, Mère d’Amani, 43 ans, Célibataire, Langui-kouadiokro.

L'attribution du nom au nouveau-né par ordre de naissance participe chez les Akan à son bien être social et sanitaire. Ce bien être est créé par la mobilisation des ressources familiales, environnementales pour la consécration des rituels, des autels et cultes d'adoration aux divinités. En retour, ces divinités se doivent de veiller sur la santé des enfants sacrés en les préservant des maladies dues au sorts, à la sorcellerie, aux mauvais esprits.

\section{Discussion}

La position ordinale de naissance de l'enfant dans la famille donne lieu à un ensemble de pratiques traditionnelles. Les groupes ethniques Abouré et Baoulé, dans l'application de la tradition, déterminent la catégorie d'enfants qui ont droit à un traitement spécial. Dans les deux groupes ethniques, c'est à partir du troisième enfant que commence l'application des soins et rituels. Les soins et les rituels constituent un ensemble de pratiques thérapeutiques que les communautés traditionnelles mettent en place pour une cohésion sociale, une intégration de ses membres et une bonne santé pour les enfants. Certains auteurs ayant écrit sur la question du rang de naissance de l'enfant soutiennent comme Collard que : «l'ordre de naissance fournit chez certains groupes un système de nomination correspondant à une hiérarchisation stricte des relations entre frères et sœurs d'une même mère » (Collard, 1973). Pour Collard, l'ordre de naissance part de l'aîné au benjamin de la famille tout en mettant en relief le respect et l'obéissance qui doivent exister entre les enfants en fonction de leur position. Tay ajoute que : «le rang de la naissance de chaque enfant intervient dans la détermination de son nom de référence ou d'appel » (Tay, 1987). À travers la pensée de Tay, il faut dire que les groupes Abouré et Baoulé appellent les enfants en fonction du rang de naissance. Chez les Abouré les noms sont les suivants : Missan, Massan, Aprâh ou Nindin, Assouan, Yamouké, Niangoran ou Yangra, Obrou et N'Ta. Chez les Baoulé ce sont: Hissan, N'Dri, Niamkè, N'Goran, Brou, Loukou, Djanhan et N’Da. Dumas-Champion va au-delà de l'idéologie de Tay en affirmant que : "nommer en imposant un nom : cet acte relève du langage performatif, au sens où il crée une situation nouvelle; le nom une fois imposé et proclamé publiquement, voici que le petit être de chair nommé devient quelqu'un de reconnu : il est intégré dans la famille et la société » (Dumas-Champion, 2002). Renchérissant, Haxaire affirme que: "Les préventions rituelles, l'imposition du nom contribuent à la protection du nouveau-né et à son inscription dans le monde » (Haxaire, 2007). Les deux auteurs parlent de l'intégration de l'enfant au sein de la société avec le nom comme premier facteur d'intégration de l'enfant dans sa famille et sa communauté. L'attribution du nom à l'enfant se fait donc chez tous avant 
l'accomplissement d'un quelconque rituel. Toute attribution du nom par rang de naissance distingue les enfants sacrés des autres enfants par leur statut spécial accompagné de soins et de rituels.

Les deux groupes ethniques Abouré et Baoulé considèrent un enfant sacré en fonction de sa position ordinale dans la famille et aussi après l'accomplissement des cérémonies qu'implique leur position. La tradition de chaque communauté donne aux enfants sacrés des droits et devoirs qui permettent leur intégration dans les familles et les communautés à travers des soins et rituels nécessaires pour leur bien-être et donc les mettre à l'abri de certaines pathologies. Selon les Abouré, un enfant est reconnu comme sacré par le collier qu'il porte ; ceci rend le port du collier nécessaire pour deux raisons principales. La première est que le port du collier permet de distinguer non seulement les enfants sacrés entre eux mais aussi au milieu d'autres enfants. La seconde raison rend compte de la pleine adhésion des parents aux us et coutumes de la communauté et fait remarquer leur intégration et celui de leurs enfants par la soumission à l'attribution du nom et des cérémonies à propos (Bonnet \& Pourchez, 2007; Pierre, 2000). En pays Abouré, faire porter aux enfants sacrés le collier correspondant à leur nom permet de leur éviter les pathologies telles que le corps chaud, la paralysie, les problèmes d'yeux, etc. Pour tout cela, du $7^{\text {ème }}$ au $10^{\text {ème }}$ enfant, les parents bâtissent en plus un autel dans la cour par le planting d'un arbre appelé « Assélé » symbole matériel de la présence en ces lieux d'un enfant sacré. À Assélé, il revient de veiller sur la santé et le bien-être de l'enfant ; c'est aussi une manière d'intégrer l'enfant dans sa communauté et lui permettre de tisser des liens sociaux avec son milieu naturel. Ce que reconnaissent Bonnet et Pourchez : "Il ne s'agit pas seulement d'assurer à l'enfant les conditions de sa survie et de sa croissance, mais aussi de l'inscrire dans une famille, dans un lignage, dans une communauté » (Bonnet \& Pourchez, 2007). Cet autre auteur ne dit pas le contraire : «les rites qui jalonnent la vie de l'enfant et souvent à intégrer la personne dans la société et à marquer différentes étapes de sa croissance véhiculent tout une idéologie » (Pierre, 2000). Leurs propos nous donnent de comprendre que l'application des soins assure aux enfants sacrés une bonne croissance, une bonne survie et les protège de toutes les maladies. En l'absence de cérémonies sacrificielles, l'enfant est victime de déséquilibre social qui peut entraîner la mort, la paralysie, des problèmes psychologiques et ophtalmologiques, ainsi que des troubles sociaux.

Chez les Baoulé N’Zikply les enfants sacrés sont ceux appartenant à la mère. Sont considérés, en pays Baoulé, comme enfants sacrés et exceptionnels les enfants suivants : Hissan, Brou, les Jumeaux et Amani. Ils sont caractériels, clairvoyants, et souvent appelés sorciers à cause de leur sixième sens très développé. Des autels sont bâtis, à leur sujet, dans la 
maison pour être adorer et les protéger. Léopold le confirme en disant que : « les enfants dénommés Hissan, Brou, $N^{\prime} D a$, sont craints et honorés. Des cultes et autels d'adoration sont installés à côté des jarres d'eau de la maison leurs sont consacrés pour prévenir des maladies et apporter le bonheur » (Léopold, 2012). Ces enfants jugés posséder une âme dangereuse est l'une des raisons pour laquelle les parents leur consacrent des autels en vue d’apaiser leur âme.

Les autres enfants sacrés comme : le $4^{\text {ème }}$, le $8^{\text {ème }}$, le $9^{\text {ème }}$, le $11^{\text {ème }}$, et le $12^{\text {ème }}$, ont chacun des bonnes âmes et sont clairvoyants. Les parents pratiquent le rituel de lavage de leur visage afin qu'ils soient protéger des maladies de tout genre. Pour cet auteur : « ces noms numérologiques sont accompagnés de cultes et autels pour prévenir des maladies et apporter le bonheur aux porteurs et aux géniteurs. » (Léopold, 2012). Ces enfants sacrés, à l'opposé de Kindôh, ont des vibrations positives qui se répercutent sur la famille par la paix, la joie, l'harmonie et le bonheur qu'ils créent. À travers le rituel de lavage du visage, les enfants reçoivent une protection spirituelle qui leur confère une bonne santé et un mieux être. S’agissant de Kindôh, troisième enfant de sexe opposé aux deux premiers, il est considéré être un enfant de mauvais augure, caractériel et capable de faire du mal à son entourage.

Si certains auteurs lient la bonne santé chez les enfants sacrés à leur ordre de naissance, aux cultes et rituels à propos, d'autres auteurs comme Akoto explique la mauvaise santé des enfants sacrés et tous les autres par l' «épuisement maternel provoqué par les grossesses successives ». Il mentionne donc que: «quant aux enfants de rang élevé, ils subissent les conséquences d'un état de santé médiocre de la mère (épuisement maternel provoqué par les grossesses successives) »(Akoto, 1985). Rakotodrabé ne dit pas le contraire de ce que dit Akoto mais clarifie sa pensée en mettant l'accent sur la mortalité infantile. Précisément, selon cet auteur : «pour une mère ayant eu une naissance de rang élevé, il y a non seulement concurrence entre ce dernier et les premiers concernant les soins, mais aussi la mère à tendance à se fier à son expérience en matière de soins en ce qui concerne le dernier ; ce qui peut augmenter le risque de mortalité » (Rakotodrabé, 1996). Akoto comme Rakotodrabé évoquent le problème de santé en s’accordant sur l'exclusion des faits rituels et cultuels avec leur supposé influence sur la santé infantile. Cependant, s'ils s’accordent sur l'exclusion des faits cultuels ou rituels, ils expriment, parlant de la problématique de santé infantile, différentes opinions. Si Akoto évoque l'épuisement maternel que provoquent les grossesses successives et en nombre important, Rakotodrabé lui parle des pratiques sanitaires des mères basées sur leur expérience dans un contexte de grossesses successives et en nombre important. Épuisement maternel pour Akoto et pratiques sanitaires materno-infantiles basées sur l'expérience pour 
Rakotodrabé sont les raisons qui justifient le taux élevé de morbi-mortalité infantile. Pour Akoto, une politique d'espacement des naissances et leur réduction serait une plausible réponse alors que dans notre cas, le contexte est favorable à la pronatalité. Au contraire, pour Rakotodrabé il serait nécessaire de faire une différence dans les soins à apporter aux enfants au regard de leur caractère spécifique et non d'une quelconque expérience acquise au nombre de naissances. Pour lui, parce que les soins maternoinfantiles diffèreraient en fonction du rang de naissance, il faut apporter des soins adaptés aux enfants en les renforçant selon leur rang de naissance. De telles pratiques diminueraient les taux de morbi-mortalité infantiles, car seuls les soins médicalement et socialement adéquats aux pathologies peuvent procurer à l'enfant une très bonne santé et un bien être.

Dans les deux communautés Abouré et Baoulé, les jumeaux sont des enfants spéciaux qui requiert des rituels, cultes particuliers et surtout plus d’attention. Chez les Nzebi au Gabon, Dupuis dit «[...] les enfants nés jumeaux sont considérés comme des génies, puissants et dangereux, qui ont choisi de venir chez les humains et ils nécessitent des cérémonies particulières et beaucoup d'attention » (Dupuis, 2007). En pays Baoulé, il s'agit de confectionner le Sèkè-sèkè et bâtir un autel dans la maison à partir de deux récipients. Ce que reconnait Kouakou en ces termes : « autrefois, les hochets servaient dans le culte des jumeaux $N^{\prime} D a$. Ils étaient le symbole de la femme en tant que mère ; c'est elle qui était la gardienne, tout comme l'autel des jumeaux. C'est elle qui était responsable de leur entretien et qui devait s'en servir en dehors de la musique » (Kouakou, 1982). En pays Abouré, le rituel au marigot et l'implantation de l'arbre Assélé dans la cour donnent lieu à une cérémonie de $N$ ’Ta. Toutes ces cérémonies sacrificielles témoignent l'attention particulière que la communauté apporte aux enfants jumeaux comme le soutient Abé : « les jumeaux sont considérés comme des êtres exceptionnels et doués d'un pouvoir occulte. À partir de ce statut particulier, ils sont l'objet d'une série de rituels. Ces rituels sont observables depuis leur naissance jusqu’à celle de leur puîné » (Abé, 2007) et Fédry qui dit que : « la naissance des jumeaux est généralement un évènement singulier nécessitant rites et précautions ; elle est marquée par l'imposition d'un nom spécial, fixé d'avance, pour chacun des jumeaux, peut-être en lien avec les précautions qu'on doit prendre à leur égard. Les enfants puînés auront eux aussi un nom spécial » (Fédry, 2009). Chez les Masa au Tchad, la naissance des jumeaux met en péril la procréation au sein de la famille parce que : «à la fois bonne et mauvaise, la naissance gémellaire s’accompagne de rites compliqués qui s’échelonnent sur une année, rites qui sont à la fois destinés à maintenir en vie les jumeaux et leurs parents, puis remettre en route de procréation des parents, fortement perturbé par ce type de naissance » (Dumas-Champion, 2002). La naissance des jumeaux est toujours un 
évènement particulier d'un peuple à un autre et chez les Masa du Tchad, la nativité gémellaire peut avoir des conséquences néfastes dans la famille en mettant fin à la procréation. La naissance gémellaire peut être à la base de psychose chez les parents. Leur attribuer un nom selon leur ordre de naissance nécessite donc un recours à des objets de santé pour une protection spirituelle et conduit à l'affirmation suivante : «l'ordre de naissance des enfants et les attitudes spirituelles qu'ils provoquent chez les Akan, témoignent d'une science qu'ils détenaient » (Allou, 2008). Les enfants sont dits sacrés à cause des rituels qui accompagnent leur position ordinale de naissance pour la sollicitation de la protection spirituelle du monde invisible sur leur santé dans ce monde visible.

En Afrique, les noms ou les prénoms ont presque chacun une histoire ; savoir l'origine de son nom ou prénom, évaluer le contexte dans lequel il a été donné c'est se plonger corps et âme dans le cœur de l'histoire familiale. Le nom ou encore le prénom traduit généralement l'identité éthique et familiale de l'enfant, voire même sa position dans la société (Zonabend, 1980). Les noms et les prénoms provenant en général de l'ethnie de laquelle on vient ont une signification précise et permettent de comprendre que dans beaucoup de familles, le choix du nom ou du prénom n'est pas anodin car il situe l'enfant et ses parents dans une époque, une catégorie sociale donnée, un groupe culturel (Eole, 2003). Le nom comme le prénom, d'après ces auteurs, rendent compte de l'identité culturelle de l'enfant et leur attribution suscite la mobilisation d'un ensemble de pratiques traditionnelles pour la survie et le bien-être de l'enfant.

Ces pratiques traditionnelles composées du port de collier, des cérémonies sacrificielles, des rituels, des consécrations d'autel et des cultes mis à contribution en pays Abouré et Baoulé améliorent le bien-être des enfants sacrés. Ainsi, selon Kouakou : " les divers objets de santé montrent que les groupes humains ont développé un système de santé pour répondre à leur besoin sanitaire » (Kouakou, 2010). Les objets de santé pensés et mis en place par les Abouré et les baoulé concourent à la bonne santé de l'enfant en le préservant des sorts, de la sorcellerie et des mauvais esprits.

\section{Conclusion}

Le nom ou le prénom des enfants sacrés provient en général de leur position ordinale dans la famille. En tant qu'enfants sacrés, ils reçoivent des soins utiles et nécessaires pour leur bien-être et leur développement au sein de la communauté. Ces soins regroupent un ensemble d'objets de santé qui sont : les colliers, les autels, les rituels et les cultes pour protéger les enfants sacrés des maladies, de la sorcellerie, et des mauvais sorts. L'attribution du nom par ordre de naissance est une forme d'intégration sociale de l'enfant. Car attribuer un nom à l'enfant c'est lui donner une identité culturelle mais 
aussi, c'est véhiculer un message ou c'est établir un lien entre son monde visible et le monde invisible. Toutes ces pratiques traditionnelles confèrent à cette catégorie d'enfants, ces enfants sacrés, une protection sanitaire préventive, une protection spirituelle et servent aussi à leur traitement curatif.

\section{References:}

1. ABÉ N. N. (2006, 2007). « La procréation, le symbolisme et la santé de la reproduction en Afrique Noire au Sud du Sahara : le cas du groupe Baoulé en Côte d’Ivoire », Université de Bouaké annéeacadémique, 875P.

2. AKOTO E. (1985) «Mortalité infantile et juvénile en Afrique : Niveaux, caractéristiques, causes et déterminants ", Département de démographie. Université Catholique de Louvaine La Neuvaine, Belgique. 273P.

3. ALLOU K. R. (2008). Onomastique, numérologique et croyances religieuses des anciens Akan, Revue ivoirienne Anthropo-Sociologie kasa Bya Kasa, ${ }^{\circ} 13$, EDUCI.

4. BONNET D. \& POURCHEZ L. (2007). Du soin au rite dans l'enfance, Editions ERES, 320P.

5. BROMBERGER C. (1982). Pour une analyse anthropologique des noms de personnes: in Langages, 16, $\mathrm{N}^{\circ} 66$, PP.103-124 : www.persée.fr/issue/igge-0458-726X-1982-num-16-66.

6. COLLARD C. (1973). Les 'noms-numéros' chez les Guidar, L'Homme 13 (3), juillet-septembre, P.P. 45-99.

7. DUMAS-CHAMPION F. (2002). La place de l'enfant dans la vie rituelle des Masa (Tchad) in Journal des africanistes, volume 72, $\mathrm{N}^{\circ} 1$, PP. 73-103.

8. DUPUIS A. (2007). 'Rites requis par la naissance, la croissance et la mort des jumeaux. Leur aménagement dans le monde moderne. Le cas des Nzebi du Gabon' in Du soin au rite dans l'enfance, Editions ERES, 2007, P.P. 255-280 ; 320P.

9. EOLE Julie (2003). Comment écrire tous ces prénoms?: http://www.irdp.ch/activites eole/annexe doc/annexe doc 11.pdf.

10. ERNY P. (2000). l'enfant dans la pensée traditionnelle de l'Afrique, l'Harmattan, le livre Africain, Paris, 200P.

11. FEDRY J. (2009). "'le nom, c'est l'homme'. Données africaines d'anthropolonymie », L’Homme 3/2009 (n¹91), P.P. 77-106, URL : www.cairn.info/revue-l-homme-2009-3-page-77.htm.

12. HAXAIRE C. (2007). " les façonnages du corps » in Du soin au rite dans l'enfance de Doris Bonnet et Laurence Pourchez, Editions érès, PP. 24-26. 
13. KASONGO K. (1988). Significations socioculturelles et psychologiques des noms individuels Ikela, in Journal des africanistes, tome 58, fascicule 2. PP. 143-151: http://www.persee.fr/doc/jafr_0399-0346_1988_num_58_2_2269.

14. KOUAKOU B. J-P. (2010). La santé infantile dans la puériculture africaine: le cas des Akan de Côte d'Ivoire : www.ethnographiques.org/IMG/pdf/ article, 12P.

15. KOUAKOU K. (1982). La musique du n'da, culte des jumeaux chez les baoulé de Dimbokro (Côte d’Ivoire), D.E.S.M, Université Français Rabelais, Tours, (Mémoire d'ethnomusicologie), 100P.

16. LÉVI-STRAUSS C. (1962). La pensée sauvage, 1962, Paris, Plon, $240 \mathrm{P}$.

17. MBITI, J. S. (1971). New Testament Eschatology in an African BackGround. Oxford, Oxford University Press.

18. SIMBANANIYE Léandre (2005). Les noms de personnes au Burundi in: Anthropologie et Sociétés, volume 29, N²1, PP. 167-181.

19. TAY A. K. B. (1987). Les problèmes de socialisation de l'enfant dans le milieu familial traitant, entre autre, de l'éducation traditionnelle et du travail de l'enfant au sein et hors de la famille ; Organisation des Nations Unies pour l'éducation, la Science et la Culture. DEV.87/MS/30, 23P.

20. VAN Gennep Arnold (1981). Les rites de passage. Etude systématique des rites, Paris, Picard, 1981, 320P.

21. YAO Yao Léopold (2012). Anthroponymie et Santé Infantile en Afrique in exemple des communautés ethniques de la Côte d'Ivoire, European Scientific journal, December vol.8, $\mathrm{N}^{\circ} 29,15 \mathrm{P}$.

22. ZONABEND Françoise (1980). "Le nom de personne » in L'Homme 20

(4) :

$7-23$; https://quartierlatin1.wordpress.com/2015/09/10/la-place-du-prenomdans-leducation-traditionnnelle-africaine-cas.d: La place du prénom dans l'Education traditionnelle Africaine: cas des Mina d'ANEHO (Togo). 